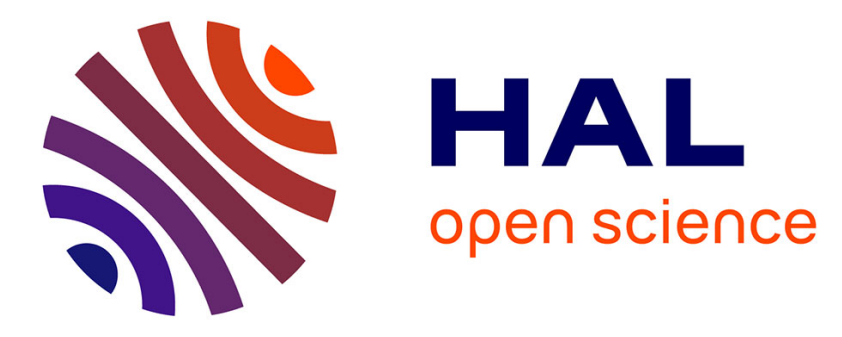

\title{
Current investigations on tritiated dust and their impact on tokamak safety
}

Christian Grisolia, François Gensdarmes, Samuel Peillon, Grégoire Dougniaux, Elodie Bernard, Adrien Autricque, Grégory Pieters, Bernard Rousseau, Sophie Feuillastre, Sébastien Garcia-Argote, et al.

\section{To cite this version:}

Christian Grisolia, François Gensdarmes, Samuel Peillon, Grégoire Dougniaux, Elodie Bernard, et al.. Current investigations on tritiated dust and their impact on tokamak safety. Nuclear Fusion, 2019, 59 (8), pp.086061. 10.1088/1741-4326/ab1a76 . hal-02159313

\section{HAL Id: hal-02159313 https://hal.science/hal-02159313}

Submitted on 18 Jun 2019

HAL is a multi-disciplinary open access archive for the deposit and dissemination of scientific research documents, whether they are published or not. The documents may come from teaching and research institutions in France or abroad, or from public or private research centers.
L'archive ouverte pluridisciplinaire HAL, est destinée au dépôt et à la diffusion de documents scientifiques de niveau recherche, publiés ou non, émanant des établissements d'enseignement et de recherche français ou étrangers, des laboratoires publics ou privés. 


\section{Current investigations on tritiated dust and their impact on tokamak safety}

To cite this article before publication: Christian Grisolia et al 2019 Nucl. Fusion in press https://doi.org/10.1088/1741-4326/ab1a76

\section{Manuscript version: Accepted Manuscript}

Accepted Manuscript is "the version of the article accepted for publication including all changes made as a result of the peer review process, and which may also include the addition to the article by IOP Publishing of a header, an article ID, a cover sheet and/or an 'Accepted Manuscript' watermark, but excluding any other editing, typesetting or other changes made by IOP Publishing and/or its licensors"

This Accepted Manuscript is @ 2019 IAEA, Vienna.

During the embargo period (the 12 month period from the publication of the Version of Record of this article), the Accepted Manuscript is fully protected by copyright and cannot be reused or reposted elsewhere.

As the Version of Record of this article is going to be / has been published on a subscription basis, this Accepted Manuscript is available for reuse under a CC BY-NC-ND 3.0 licence after the 12 month embargo period.

After the embargo period, everyone is permitted to use copy and redistribute this article for non-commercial purposes only, provided that they adhere to all the terms of the licence https://creativecommons.org/licences/by-nc-nd/3.0

Although reasonable endeavours have been taken to obtain all necessary permissions from third parties to include their copyrighted content within this article, their full citation and copyright line may not be present in this Accepted Manuscript version. Before using any content from this article, please refer to the Version of Record on IOPscience once published for full citation and copyright details, as permissions will likely be required. All third party content is fully copyright protected, unless specifically stated otherwise in the figure caption in the Version of Record.

View the article online for updates and enhancements. 


\title{
Current investigations on tritiated dust and their impact on tokamak safety
}

\author{
C. Grisolia ${ }^{1}$, F. Gensdarmes ${ }^{2}$, S. Peillon ${ }^{2}$, G. Dougniaux ${ }^{2}$, E. Bernard ${ }^{1}$, A. Autricque ${ }^{1}$, G. Pieters ${ }^{3}$, \\ B. Rousseau ${ }^{3}$, S. Feuillastre ${ }^{3}$, S. Garcia-Argote ${ }^{3}$, O. Carvalho ${ }^{3}$, V. Malard ${ }^{4}$, I. George ${ }^{4}$, \\ L. Lebaron-Jacobs ${ }^{4}$, T. Orsiere ${ }^{5}$, C. Uboldi ${ }^{5}$, J. Rose ${ }^{6}$, M Sanles Sobrido $^{6}$, D. Lambertin ${ }^{7}$, D. Vrel ${ }^{8}$, \\ C. Decanis ${ }^{9}$, K. Liger ${ }^{9}$ \\ ${ }^{I}$ CEA, IRFM, Saint Paul-lez-Durance, France \\ ${ }^{2}$ Institut de Radioprotection et de Sureté Nucléaire (IRSN), Gif-sur-Yvette, France \\ ${ }^{3}$ CEA, JOLIOT, Gif-sur-Yvette, France \\ ${ }^{4}$ CEA, BIAM, UMR7265 CEA/CNRS/Aix Marseille Univ., Saint Paul-lez-Durance, France \\ ${ }^{5}$ Aix Marseille Univ., Avignon Université, CNRS, IRD, IMBE, Marseille, France \\ ${ }^{6}$ Aix Marseille Univ., CNRS, IRD, Collège de France, INRA, CEREGE, Aix en Provence, France \\ ${ }^{7} C E A, D E N$, Bagnols-sur-Cèze, France \\ ${ }^{8}$ LSPM, Université Paris 13, Sorbonne Paris Cité, CNRS UPR 3407, Villetaneuse, France \\ ${ }^{9} \mathrm{CEA}, \mathrm{DEN}$, Saint Paul-lez-Durance, France \\ Corresponding author:christian.grisolia@cea.fr
}

\begin{abstract}
During ITER operation, due to plasma wall interaction, particles/dust will be created with sizes ranging from nanometer to tens of micron. The dust properties especially their ability to be covered by a thin oxide electrostatic insulating layer and their surface topology deeply affect their tritium inventory. Consequently, physico-chemical properties specific to tritiated tungsten particles and consequence on particles behavior in the facility and environment must be carefully assessed. For size relevant tungsten particles, the measured tritium inventory is $\sim 10 \mathrm{GBq} / \mathrm{g}$. However, it varies with the particle specific surface area (SSA). Due to tritium beta decay and to the oxide-insulating layer, dust exhibits a positive electrostatic self-charging. For a $5 \mu \mathrm{m}$ particle in diameter with a 10 $\mathrm{GBq} / \mathrm{g}$ tritium inventory, self-charging rate could lead to $5.510^{4}$ elementary electric charges per day. These electrostatic properties could change the adhesion of dust on walls. In case of a single particle, the adhesion will be reinforced due to image and dielectric forces. However, if the tritiated particle is part of an aggregate, the adhesion remains unknown. Due to the limited free path of the $\beta$ emission in material, the tritium inventory carried by airborne particles cannot be measured in real time by conventional continuous radioactive aerosols monitors (CAM's) and a new measurement strategy is needed for atmospheric survey in workplace and exhaust of facility. Toxicity studies dealing with exposure to untritiated/tritiated tungsten particles of $100 \mathrm{~nm}$ have been undertaken. It was shown that these particles are rapidly dissolved in biologic media. Finally, after collection, dust must be confined to avoid their spreading into the environment. Different technical solutions are presented in the paper.
\end{abstract}




\section{INTRODUCTION}

During operation of the International Thermonuclear Experimental Reactor (ITER), plasma interacts with the plasma facing components (PFCs) through various physical processes. These give birth to particles from nanometer to tens of microns in size that are called dust grains in the fusion community. Depending on the plasma wall interaction processes at play, different types of dust will be created, from almost spherical particles induced by high heat flux deposition on metal surfaces (unipolar arcs, ELMs, disruptions) to fractal ones created by accretion in the edge of high density/long pulse plasma discharges. The metallic dust properties, especially their ability to be covered by an electrostatic insulating oxide layer and their surface topology, deeply affect their tritium inventory, their chemical reactivity and their capability to become airborne and to be released out of the tokamak vacuum vessel.

This paper discusses dust properties linked with safety and radioprotection open issues. These aspects are synthetically presented in Fig. 1.

First, experimental results on dust tritium inventory obtained for $W$ material are recalled. Extrapolations from deuterium trapping in massive beryllium samples are then established.

Second, the consequences of the natural dust charging induced by tritium radioactive decay on dust adhesion and on aerosol creation in case of Loss Of Vacuum Accident (LOVA) are investigated. The behaviour of an isolated particle is compared to a dust aggregate in terms of dust adhesion/suspension.

The limited free path of the $\beta$ electron in materials used in fusion devices has major consequences on the aerosol tritium inventory measurement in real time. This is addressed in the third part of this paper.

When an aerosol is created, workers can be contaminated by inhalation of tritiated particles. Our team has been involved for years now on the evaluation of the in-vitro cyto and genotoxicity of tritiated and untritiated $W$ particles on lung cells. Summarized results are presented in the fourth part of this paper. Finally, we will address the dust waste management open issues and the solution that we decided to promote.

\section{DUST TRITIUM INVENTORY}

Study of tritium trapping in W dust material has been initiated by our team in 2011 [1]. It has been shown that the dust tritium inventory is two to three orders of magnitude higher than in massive material. It ranges from 1 to $20 \mathrm{GBq} / \mathrm{g}$ depending on the Specific Surface Area (SSA) of the particles. The higher the SSA, the larger the measured tritium inventory. When extrapolated to $1000 \mathrm{~kg}$ of W dust (corresponding to the dust safety limit) only $\sim 50 \mathrm{~g}$ of tritium is trapped in such powdery material [2]. That is well below the tritium safety limit in vessel of ITER (1000 g).

No comparable studies exist for beryllium (Be) powder. As a consequence, estimation of tritium (T) content per $\mathrm{kg}$ of Be powder in the ITER vacuum vessel requires extrapolation of existing studies conducted on deuterium (D) trapping in Be massive materials $[3,4]$. One can thus estimate the tritium content in the ITER vacuum vessel per kilogram of beryllium powder. The $D / B e$ atomic ratio varies from 0.01 up to 0.1 depending on the deuterium loading conditions and on the beryllium target temperature. By assuming that the $\mathrm{T} / \mathrm{Be}$ ratio is comparable to the $\mathrm{D} / \mathrm{Be}$ one, the tritium limit in the vacuum vessel is reached for $600 \mathrm{~kg}$ of $B e$ dust for $T+D / B e=0.01$ and for $60 \mathrm{~kg}$ for $T+D / B e=0.1$. This last value corresponds to a rather strong tritium activity of $\sim 6 \mathrm{TBq} / \mathrm{g}$.

These preliminary estimations emphasize the necessity to collect and analyze particles produced during the ITER lifespan. Moreover, the collection of dust produced in current operating machines is 
necessary in order to prepare the ITER operation, but not sufficient. The operating domain of ITER will be different from current tokamaks giving birth to dust with rather different properties. In dusty plasmas [5], the erosion of a $\mathrm{W}$ target in high density and low temperature plasma leads to the creation of large quantities of fractal dust with high SSA. These conditions are not observed in current tokamaks but would be reached in detached ITER plasma regime. As a consequence, extrapolation from current tokamak dust collection could be unrepresentative.

Finally, it has to be noticed that if some experiments are undertaken to study tritium inventory in tungsten dust, no comparable effort is put on beryllium powder. Such lack of experimental results is detrimental for ITER safety prediction.

\section{TRITIATED DUST ELECTROSTATIC PROPERTIES}

\subsection{Electrostatic properties of tungsten metallic dust}

Metallic tungsten dust particles are covered by a thin oxide layer of 4-6 $\mathrm{nm}$ that induces insulating electrostatic properties. The experimental set-up used by Peillon et al. [6] to study dust suspension induced by an applied electric field consists of two circular stainless steel electrodes that are arranged in parallel and separated by a $4 \mathrm{~mm}$ gap. The top electrode is hooked to a high voltage source whereas the bottom electrode, which includes a central extraction orifice, is grounded. A monolayer circular deposit of particles, $4 \mathrm{~cm}$ in diameter, is spread out on the top electrode. An increasing electric field (E) from 0 up to $27.5 \mathrm{kV} / \mathrm{cm}$ is then applied by steps of $2.5 \mathrm{kV} / \mathrm{cm}$ in ambient atmospheric conditions. For each applied $\mathrm{E}$, the detached particles are pumped out and transported by a radial airflow through the central extraction hole connected to an optical particle counter (OPC) that measures the size-resolved particle number concentration. A schematic representation of this set-up is depicted in Fig. 2.

The results obtained for different types of powder (silver, tungsten and aluminum oxide $\mathrm{Al}_{2} \mathrm{O}_{3}$ ) are displayed in Fig. 3 where the cumulative fractions of particles detached and counted by the OPC are plotted with respect to the electric field strength. The data are normalized thanks to the total amount of particles detached by the maximum electric field achievable in the experimental set-up (i.e. $27.5 \mathrm{kV} / \mathrm{cm}$ ).

This experiment reveals that the presence of a thin oxide layer on the tungsten particle surfaces alters their electrical conductivity by shifting their properties from conductive (silver behavior) to insulating (dielectric) material (aluminum oxide behavior). Consequently, tungsten particles will experience an accumulation of electric charges due to tritium $\beta$-decay even when deposited on grounded metallic surfaces. The next section presents the evaluation of the particle self-charging rate according to the tritium $\beta$-decay. One should notice that this work provides data for electrostatic charge build-up, which is a necessary input to assess a final charge equilibrium state taking also account for the neutralization processes due to the dielectric properties of the materials in contact or ion fixation. These neutralization processes are not studied in the framework of this paper.

\subsection{Tritium decay and consequence on dust electrostatic self-charging}

Tritium is a radioactive isotope of Hydrogen. Its half-life is 12.3 years. During the tritium decay, a $\beta$ emission is observed (mean energy of $5.7 \mathrm{keV}$ ) along with ${ }^{3} \mathrm{He}^{+}$production.

For the mean energy of $5.7 \mathrm{keV}$, the $\beta$ maximum path ( $\mathrm{Lm})$ can be calculated [7] and gives $\mathrm{Lm}=43$ $\mathrm{nm}$ in the case of $\mathrm{W}$ and $450 \mathrm{~nm}$ for Be. The $\mathrm{Lm}$ limited value has several consequences. First, for large particles, not all the $\beta$ emission escapes from the material. For $W$, only an external layer of 
$\sim 100 \mathrm{~nm}$ contributes to the resulting positive dust charging. As an example, taking into account the full spectrum energy of $\beta$ decays of tritium, the probability of electron escape from a $100 \mathrm{~nm}$ thickness layer is $20 \%$. It has to be pointed here that the insulating properties of the metallic dust demonstrated in paragraph 3.1 will insure a quasi-permanent positive charge in the particles.

Full Monte Carlo calculations [8] of the self-charging rate of $W$ particles with $10 \mathrm{GBq} / \mathrm{g}$ tritium inventory have been performed. The charging rate is presented in Fig. 4 as a function of the particles diameter.

The continuous line represents the electrical self-charging rate of a particle where the tritium is homogeneously distributed in the material while the dashed one concerns a particle where the tritium is distributed in a surface layer having a thickness of $100 \mathrm{~nm}$. As expected by the boundary conditions applied, the two self-charging rates differentiate (discrepancy above $10 \%$ ) when the particle diameter reaches $400 \mathrm{~nm}$.

For example, a single tungsten particle of $5 \mu \mathrm{m}$ in diameter with homogeneous distribution of tritium will exhibit a self-charging rate of $5.5 \times 10^{4}$ positive charges per day.

Nevertheless, tritium is only a part of radionuclides to take into account in radioactive dust issues in fusion tokamak and self-charging processes. Other radionuclides are present in fusion tokamak coming from activation by neutron fluxes. Usually the calculations and study made on the activation due to neutron fluxes in the framework of fusion machine deal with dose rate and are mainly focused on activation products that are gamma emitters.

It should be noticed that gamma decay and high radiation level does not lead to particles selfcharging rate like one for beta or alpha decay. The case of gamma ray with high dose rate effect upto $7 \mathrm{kGy} / \mathrm{h}$ on self-charging was investigated by Gensdarmes et al. [9, 10]. Self-charging due to gamma decay is a very weak process due to the poor probability of photon interactions (photoelectric absorption or Compton scattering) with particles. This mainly comes from the small length path available for photon interactions in micron size particles.

Let the case of beta emitters radionuclide produced by neutron activation be examined. There are few papers in the literature dealing with quantitative assessment of dust contamination for neutron activation scenario related to the ITER operational condition. Nevertheless, the paper by Saito et al. [11] reports input data to assess self-charging rate due to activation products in comparison to Tritium. In particular, the authors mention a list of radionuclides induced by neutron activation and their specific activity $(\mathrm{Bq} / \mathrm{g})$ in a comparable manner than to the specific activity value we determine for tritium in tungsten particles.

The main beta emitter radionuclides to be considered [11] for self-charging are two isotopes of tungsten: ${ }^{187} \mathrm{~W}$ and ${ }^{185} \mathrm{~W}$. The other beta emitter radionuclides of the list, Ta and Re, have low specific activities that are one to three orders of magnitude below tungsten isotopes and they have very short half-life. The ${ }^{60} \mathrm{Co}$, have a medium half-life but a specific activity four to five orders of magnitude below those of tungsten isotopes.

The specific activity values are $10^{11} \mathrm{~Bq} / \mathrm{g}$ for ${ }^{187} \mathrm{~W}$ is (half-life 23.7 hours) and $3.710^{10} \mathrm{~Bq} / \mathrm{g}$ for ${ }^{185} \mathrm{~W}$ (half-life 75 days). Besides, specific activity value for ${ }^{3} \mathrm{H}$ in tungsten particle is up to $10^{10} \mathrm{~Bq} / \mathrm{g}$ (Grisolia et al., [2]) while its half-life is 12.3 years. Consequently, according to these data, on short time period (< some days), the radionuclide ${ }^{187} \mathrm{~W}$ will dominate electrostatic self-charging; for intermediate term (some days to some months) tungsten isotopes ${ }^{185} \mathrm{~W}$ and ${ }^{187} \mathrm{~W}$ may contribute in equal part like tritium to self-charging. However, on long term period (> some months) the tritium beta decay will dominate electrostatic self-charging. 
It is obvious that this conclusion depends on the neutron activation scenario. The data reported in [11] for activation products comes from the ITER Preliminary Safety Report.

All these considerations shows that beta decay of tritium appears as a key component of electrostatic properties of dust as soon as trapped activity relies on such high level as $10^{10} \mathrm{~Bq} / \mathrm{g}$.

\section{CONSEQUENCES ON THE DUST ADHESION}

\subsection{Adhesion of an uncharged particle}

The adhesion of an uncharged particle relies mainly on the van der Walls force (for the sizes of particles considered, the gravity is negligible). The adhesion force depends on different parameters such as the Hamaker constant of materials into contact, the distance of closest approach between the materials, the roughness of the contacting surfaces, which are difficult to estimate.

Fortunately, adhesion forces can be directly assessed using the Atomic Force Microscope (AFM) technique. Details on the technique and experimental procedure can be found in [12]. Adhesion force measurements have been realized with a Multimode 8 (Bruker) AFM in PeakForce Quantitative Nano-Mechanical mode (PF-QNM) in environmental conditions. The measurements were realized between tungsten spherical particles with different sizes glued onto tip-less CP-FM (Colloidal Probe for Force Modulation) cantilevers and three different tungsten surfaces with various roughnesses. Samples were cleaned by successive ultrasonic baths of acetone and ethanol and dried before being mounted in the AFM. The results obtained are summarized in Fig. 5 where the W/W adhesion force is plotted as a function of the particle radius for different root mean square (rms) surface roughness.

As it can be seen in Fig. 5, the adhesion force decreases with the rms roughness of the substrate and becomes quasi-independent on the particle size for high values of roughness i.e. $\approx 700 \mathrm{~nm}$. Moreover, the adhesion force on a relevant $W$ tokamak surface $(\mathrm{rms} \sim 712 \mathrm{~nm}$ ) is two orders of magnitude lower than on a smooth mirror polished tungsten surface.

\subsection{Adhesion of a tritiated particle}

As we have seen, metallic particles ( $\mathrm{W}$ or Be) are covered by an insulating oxide layer. When such a particle is charged, an image force appears as presented schematically in Fig. 6 for a W dust covered by a $\mathrm{WO}_{3}$ oxide layer.

This electrostatic image force will reinforce the van der Waals force and the overall adhesion process. As an example and considering that the oxide layer acts as a perfect insulator, it will take $\sim 14$ hours to get an image force equivalent to the van der Waals one i.e. $\sim 10 \mathrm{nN}$ for a particle of 5 $\mu \mathrm{m}$ of diameter and $10 \mathrm{GBq} / \mathrm{g}$ of homogeneous tritium inventory.

However, in a tokamak like ITER, dust will fall towards the bottom of the machine and pile up. In that case, the consequences of self-charging could be rather different. Indeed, at the top of a pile, particles could undergo an electric force from positively charged particles located below them. This situation can strongly affect the particles adhesion which can induce an easiest particles/aggregate suspension in case of a LOVA and jeopardize the prediction of aerosol creation.

Our team has started studies on the adhesion of isolated tritiated particles. Comparable studies must now take place for piled-up particles in order to get reliable information on this open issue. 


\section{MEASUREMENT OF TRITIUM CARRIED BY AEROSOLS}

As already mentioned, due to the low $\beta$ path in the material only a small part of the tritium activity can be measured out of a tritiated particle. Fig. 7 represents Monte Carlo calculations of electron escape probability for spherical tritiated tungsten particle, taking into account the full energy spectrum of tritium $\beta$ decays. For micrometer spherical tungsten particles, these results show that only the $\beta$-decay of tritium located in a surface layer of $\sim 100 \mathrm{~nm}$ can lead to an electron escape probability from the particle above $20 \%$ and thus be detected. As a consequence, a real time tritium measurement by an ionization chamber as recommended by the Standard IEC 60761-5 [13] will lead to an underestimated value of the aerosol tritium inventory.

Usually, at the laboratory scale, the total tritium inventory in dust is measured by full dissolution of the particles followed by liquid scintillation counting. This is a reliable diagnostic but this procedure cannot be applied in real time. Moreover, this method fails to distinguish tritium carried by particles from the gaseous form in a simple way.

This leads to another open issue dealing with atmospheric survey of workplace area and exhaust that must be addressed carefully: to develop a methodology/diagnostic able to accurately measure the total tritium content in tritiated aerosol.

\section{TOXICITY STUDIES}

In case of LOVA, an aerosol can be created. To prevent contamination to the environment and/or to people supervising the cleaning operations, High Efficiency Particulate Air (HEPA) filters are used. However, these filters have a lower retention capability for particles with aerodynamic diameters in the $100-300 \mathrm{~nm}$ range $[14,15]$.

We therefore studied the consequences of inhaling small particles that can reach the human inner lung. As we have seen above, on long term period (> some months) the tritium beta emission will dominate and the toxicity studies we undertaken were focused on tritiated particles.

Different topics were thus addressed such as the behaviour of particles and embedded tritium in biologic media or the in vitro toxicity of these tritiated particles.

The work was focused on 100-200 nm tritiated and non-tritiated W particles. From Fig 8, we can state that those particles were rapidly dissolved in different biologic media as saline solution, TRIS buffer and cell culture medium "lung solution". TRIS solution was used to prepare particles stock solution for in vitro studies.

A full dissolution was obtained after 2 to 60 days of exposure to $100-200 \mathrm{~nm} \mathrm{~W}$ particles depending on the dust type $[16,17]$. In addition, a full dissolution of large $W$ particles (up to $7 \mu \mathrm{m}$ of diameter) was also observed.

The consequence of the dissolution of the particles is the release of tritium into the solution. Tritium, once solubilized, will be removed quickly. Indeed, after human exposure, tritium in tritiated water form is eliminated very quickly from the body (half-life of 10 days) [18].

Toxicity of untritiated and tritjated tungsten particles has been studied on a 3D in vitro cell model of the human airway epithelium grown on air-liquid-interface, MucilAir ${ }^{\circledast}$. This model is characterized by similar morphology and functions of the normal human airway epithelium and by mucus secretion and cilia beating [19]. Epithelia were exposed for $24 \mathrm{~h}$ to tungsten nanoparticles (tritiated or not) or tritiated water, as a control. Thanks to the long shelf life of this model, cytotoxicity was studied immediately after treatment and in a kinetic mode up to 1 month after cell exposure to assess the reversibility of any toxic effect. Acute and long-term toxicities were monitored by several endpoints: 1) epithelial integrity, 2) cellular metabolic activity, 3) pro-inflammatory response, 4) mucociliary clearance, and 5) morphological modifications. Transmission Electronic Microscopy observations, 
Inductively Coupled Plasma Mass Spectrometry measurements and liquid scintillation were performed to determine tungsten and tritium human airway epithelium lung absorption as well as intracellular accumulation. In parallel, their potential toxicity was assessed on human-derived immortalized bronchial cells (BEAS-2B cells). Untritiated and tritiated tungsten particles exerted different degrees of cytotoxicity depending on the presence/absence of hydrogen on their surface. Differences were observed also in their genotoxic potential investigated by performing the centromeric cytokinesis-block micronucleus cytome test (CBMN-cyt) and the alkaline comet assay. Furthermore, DNA methylation analysis was also assessed to determine epigenetic effects on BEAS$2 \mathrm{~B}$ cells.

These observations are the first steps towards establishing future potential guidelines for LOVA and workplace safety. However, further studies are needed on W particles as well as on Be particles.

\section{TRITIATED DUST AND WASTE MANAGEMENT}

Tritiated dust will be collected in rather large quantities (hundreds of kilograms) during the ITER life. After vacuuming the ITER in vessel, they will be temporarily stored in canister.

From a safety point of view, avoiding tritiated dust spreading into the environment is a major concern. As a consequence, the storage in canister is not appropriated as a permanent storage due to its weakness. Moreover, it is needed to define the best route for ITER tritiated dust to mitigate the risk as close as possible to the source. As a consequence, it is recommended to limit the recovery or handling of dust from the canister to avoid spreading.

In this framework, after deep analysis of all the possible technical possibilities, we propose to study two solutions: (i) immobilization in a hydraulic binder and (ii) dissolution followed by immobilization in a hydraulic binder. Both solutions will mitigate the risk of spreading before grouting and prevent from any reconditioning. However, the second one requires addition of a chemical and the associated risk has to be managed in a dedicated cell.

One of the main problem that is expected is the strong reactivity of metallic powder $(\mathrm{W}, \mathrm{Be})$ when introduced in cement due to the high $\mathrm{pH}$ of the solution. This leads to hydrogen emission that must be controlled. Experiments will start in fall 2018 at CEA to study both aforementioned solutions.

\section{CONCLUSION}

During the operation of a tokamak like ITER, dust will be produced by plasma wall interaction processes. These particles will have a large set of properties due to their diverse morphologies including particles of high chemical reactivity. The dust that have been collected in current tokamaks have certain properties that cannot be definitively extrapolated to ITER. Indeed, the current tokamak operation cannot yet represent the ITER operating regime. As an example, ITER will rely on quasi detached divertor plasmas with a high density and low plasma temperature characteristics for all the shot duration. In these conditions, it has been shown in dusty plasma laboratory studies [20] that relatively small particles (diameter $<1 \mu \mathrm{m}$ ) of large SSA and consequently large chemical reactivity are created in quantity. This will probably be also the case during ITER operation. It will be thus mandatory to collect dust produced during the ITER life in order to refine our knowledge on their physicochemical properties.

The dust tritium inventory in ITER-relevant particles has been studied in the case of $\mathrm{W}$ showing that the tritium inventory varies with the SSA of the particles. The measured tritium content is $\sim 10 \mathrm{GBq} / \mathrm{g}$ for $\mathrm{W}$ micrometer-sized particles. Extrapolation from existing results on deuterium trapping in massive Be leads to huge dust tritium inventory greater than $6 \mathrm{TBq} / \mathrm{g}$. It is obvious that studies on tritium trapping in ITER relevant Be dust are urgently needed to confirm or deny this tendency.

Dust are covered by an oxide layer that is an electric insulator. Due to tritium decay this will lead to the build-up of positive charges in the particles. In case of a $\mathrm{W}$ particle of $5 \mu \mathrm{m}$ diameter with a tritium inventory of $10 \mathrm{GBq} / \mathrm{g}$, the resulting charge is more than $5.510^{4}$ positive charges per day. 
In case of an isolated particle deposited on the vessel wall, self-electrical charging will increase the dust adhesion force making aerosol creation more difficult. However, in the case of aggregates of tritiated particles, the adhesion remains unknown.

Due to limited low path of the $\beta$ emission in metallic particles, the measurement of the tritium aerosol inventory in real time remains unachieved. This points the necessity to perform R\&D to cope with this open issue.

In case of LOVA and the creation of a tritiated aerosol, particles can escape the vacuum vessel and get through the HEPA filters. We have undertaken some toxicity studies that concern inhalation of tritiated and untritiated W particles of $100-200 \mathrm{~nm}$. We have shown that these particles are rapidly dissolved in biologic media. All these studies will give useful information to define potential guidelines in case of LOVA and for occupational safety.

Finally, it has been shown that the waste management of tritiated dust needs special methodology in order to avoid any spread into the environment. This requires the development of a dust immobilization technique. After deep analysis of all the existing solutions, CEA has proposed two solutions e.g. immobilization in hydraulic binder or dissolution of dust followed by immobilization in hydraulic binder. These two solutions are currently under studies at CEA.

\section{ACKNOWLEDGEMENT}

The authors thanks Benjamin DECHENAUX and Eric DUMONTEIL from the Neutronics Laboratory of the Institut de Radioprotection et de Sûreté Nucléaire for helpful discussions about activation of tungsten compounds by neutron fluxes. 
[1] El-Kharbachi A. et al., Tritium absorption/desorption in ITER-like tungsten particles, International Journal of Hydrogen Energy, 39 (2014) 10525-10536.

[2] Grisolia C. et al, Tritium absorption and desorption in ITER relevant materials: comparative study of tungsten dust and massive samples. Journal of Nuclear Materials 463 (2015) 885-888.

[3] Doerner R. et al., Codeposition of deuterium with ITER materials, Nucl. Fusion, 49, (2009) 035002.

[4] Lahtinen A. et al, Deuterium retention in the divertor tiles of JET ITER-Like wall, Nuclear Materials and Energy 12 (2017), 655-661.

[5] Barbosa S. et al, An introduction to light extinction spectrometry as a diagnostic for dust particle characterization in dusty plasmas, J. Plasma Phys. 82 (2016), 615820403.

[6] Peillon S. et al, Mobilization of tungsten dust by electric forces and its bearing on tritiated particles in the ITER tokamak, Journal of Electrostatics, 88 (2017), 111-115.

[7] Everhart T, Hoff P, Determination of Kilovolt Electron Energy Dissipation vs Penetration Distance in Solid Materials, J. App. Phys. 42 (1971), 5837.

[8] Dougniaux G. et al, Monte-Carlo simulations of electrostatic self-charging of tritiated tungsten and beryllium particles, To be published in Proceedings of Electrostatics 2019 conference, Journal of Physics: Conference Series.

[9] Gensdarmes F., Boulaud D., Renoux A. Aerosol charging under gamma irradiation. J. Aerosol Science. Vol. 29, Suppl. 1. pp. S851-S852. 1998. https://doi.org/10.1016/S0021-8502(98)90608-5

[10] Gensdarmes F., Boulaud D., Renoux A. The electric charging of aerosols in high ionized atmosphere. AIP Conference Proceedings 534, 765 (2000); https://doi.org/10.1063/1.1361972.

[11] Saito et al, Preliminary assessment for dust contamination of ITER In-Vessel Transporter. Fusion Engineering and Design 89 (2014) 2352-2356. http://dx.doi.org/10.1016/j.fusengdes.2014.02.023

[12] Peillon S. et al, Adhesion of tungsten particles on rough tungsten surfaces using Atomic Force Microscopy, under review in Journal of Aerosol Science.

[13] IEC 60761-5, Equipment for continuous monitoring of radioactivity in gaseous effluents - Part 5: Specific requirements for tritium monitors.

[14] Vendel J. et al, Lessons learnt over 30 years of air filtration in the nuclear industry, Journal of Physics: Conference Series. 170 (2009), 012026.

[15] Huang S.H. et al, Factors Affecting Filter Penetration and Quality Factor of Particulate Respirators, Aerosol and Air Quality Research. 13 (2013), 162-171.

[16] Bernard E. et al, Design of model tokamak particles for toxicity studies: morphology and physical characterization, Fusion Engineering and design, 2018, accepted for publication.

[17] Sanles Sobrido M. et al, Oxidative transformation of Tungsten (W) submicron particles released in aqueous and biological media in case of Tokamak (nuclear fusion) Lost of Vacuum Accident (LOVA). Corrosion Science, 2018, under review.

[18] ICRP, 2016, Occupational Intakes of Radionuclides: Part 2. ICRP Publication 134. Ann. ICRP 45(3/4), 1-352.

[19] Constant S. et al, MucilAir: a novel in vitro human 3D airway epithelium model for assessing the potential hazard of nanoparticles and chemical compounds, Toxicology Letters, 2008, 180, S233.

[20] Barbosa. et al, In-situ characterization of the dynamics of a growing dust particle cloud in a direct-current argon glow discharge, 2016 J. Phys. D: Appl. Phys. 49045203. 
FIGURES

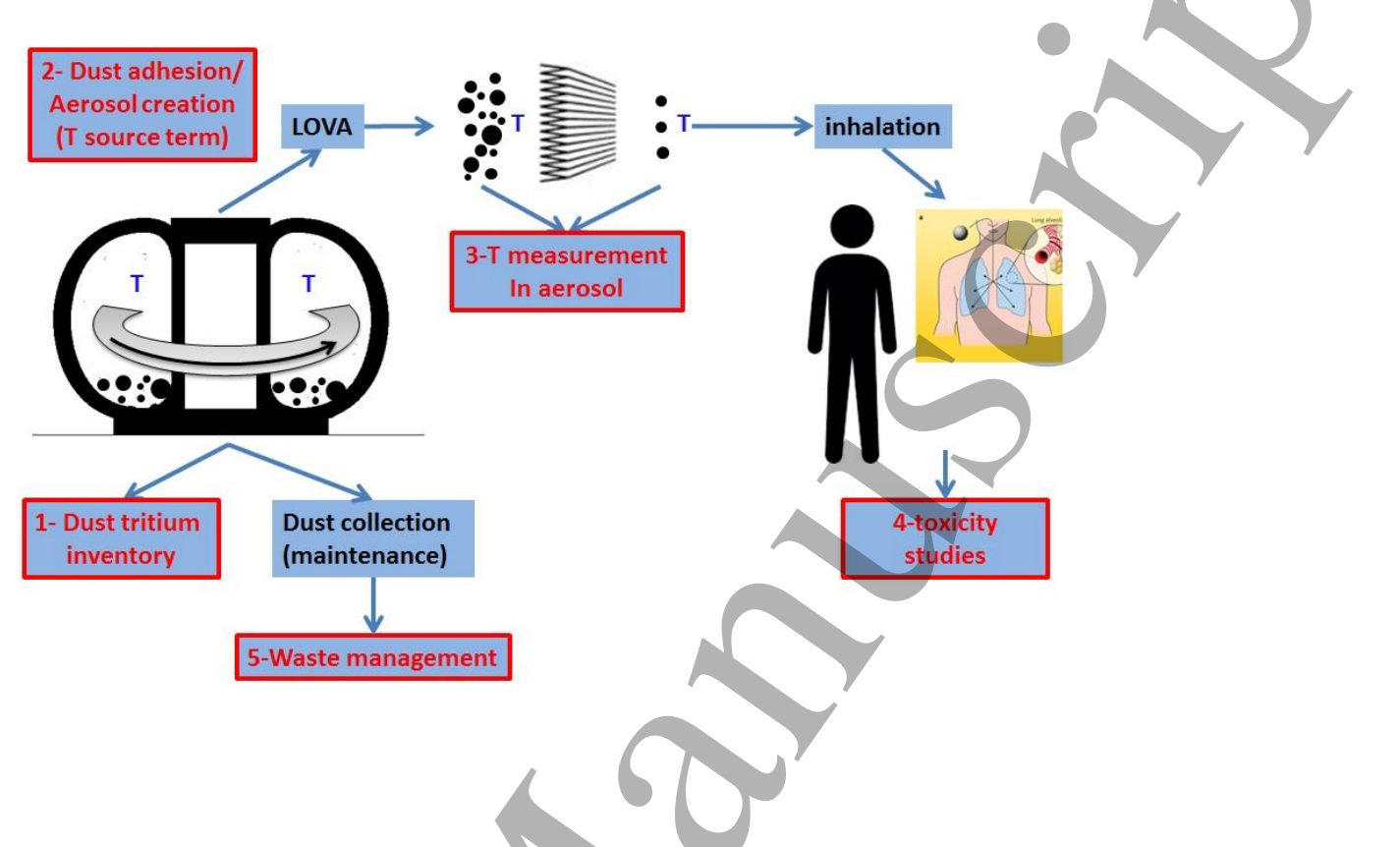

FIG. 1. Synthetic presentation of the topics covered in the paper. 


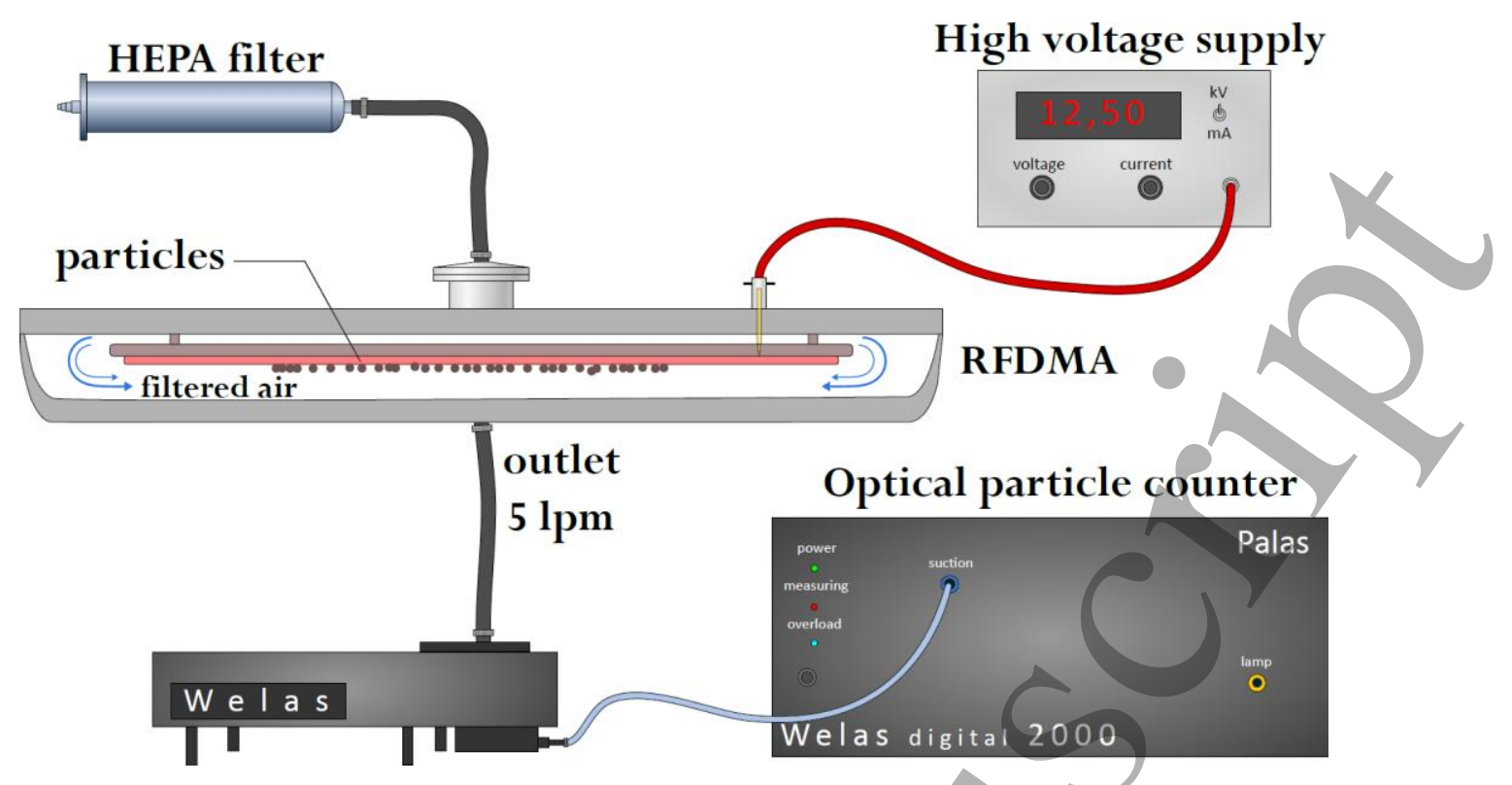

FIG. 2 Schematic representation of the experimental set-up used in [6]. RFDMA accounts for Radial Flow Differential Mobility Analyzer. 


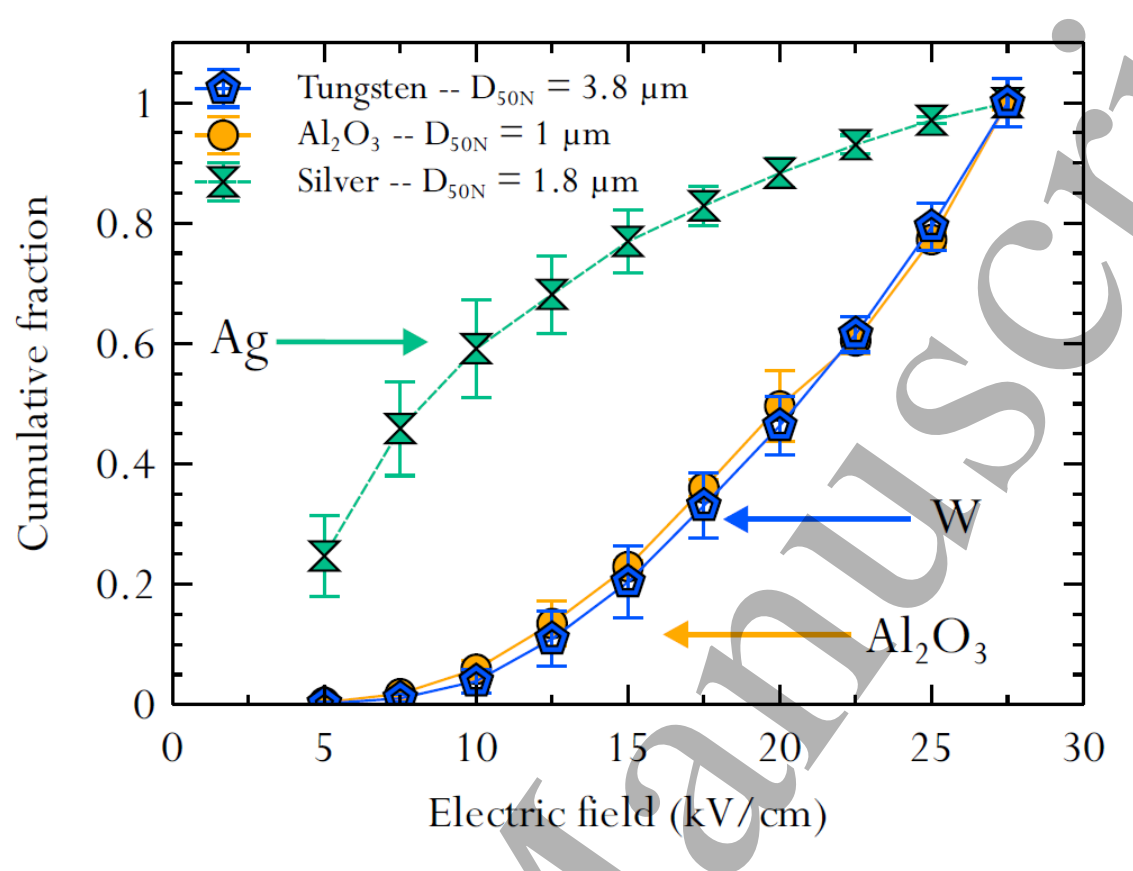

FIG. 3. Normalized cumulative number fraction of particles ( $\mathrm{W}, \mathrm{Al}_{2} \mathrm{O}_{3}$ and $\mathrm{Ag}$ ) experiencing increasing electric field strengths. 


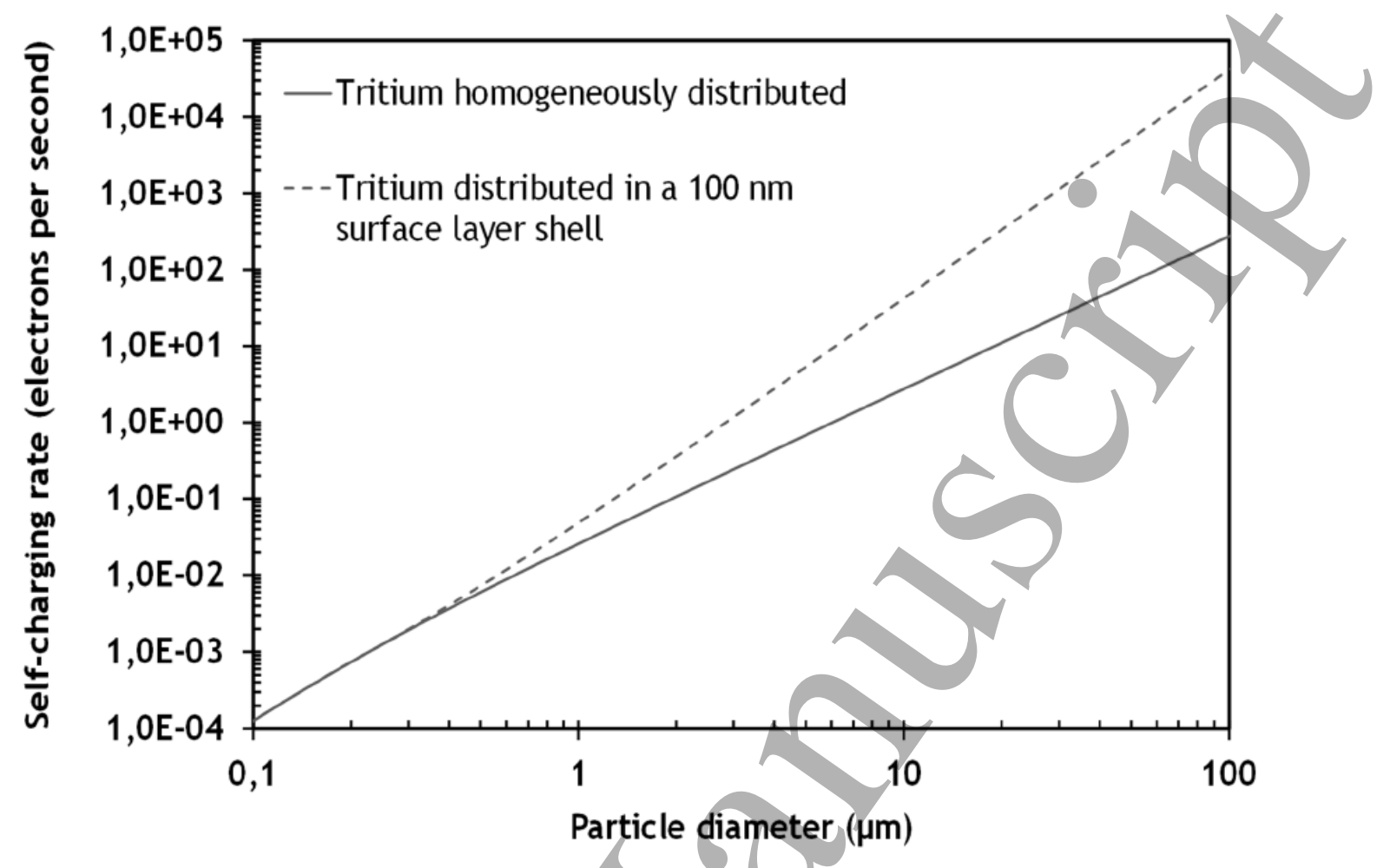

FIG. 4. Calculation of the electrical self-charging rate of tungsten particles considering a tritium inventory of $10 \mathrm{GBq} / \mathrm{g}$. 


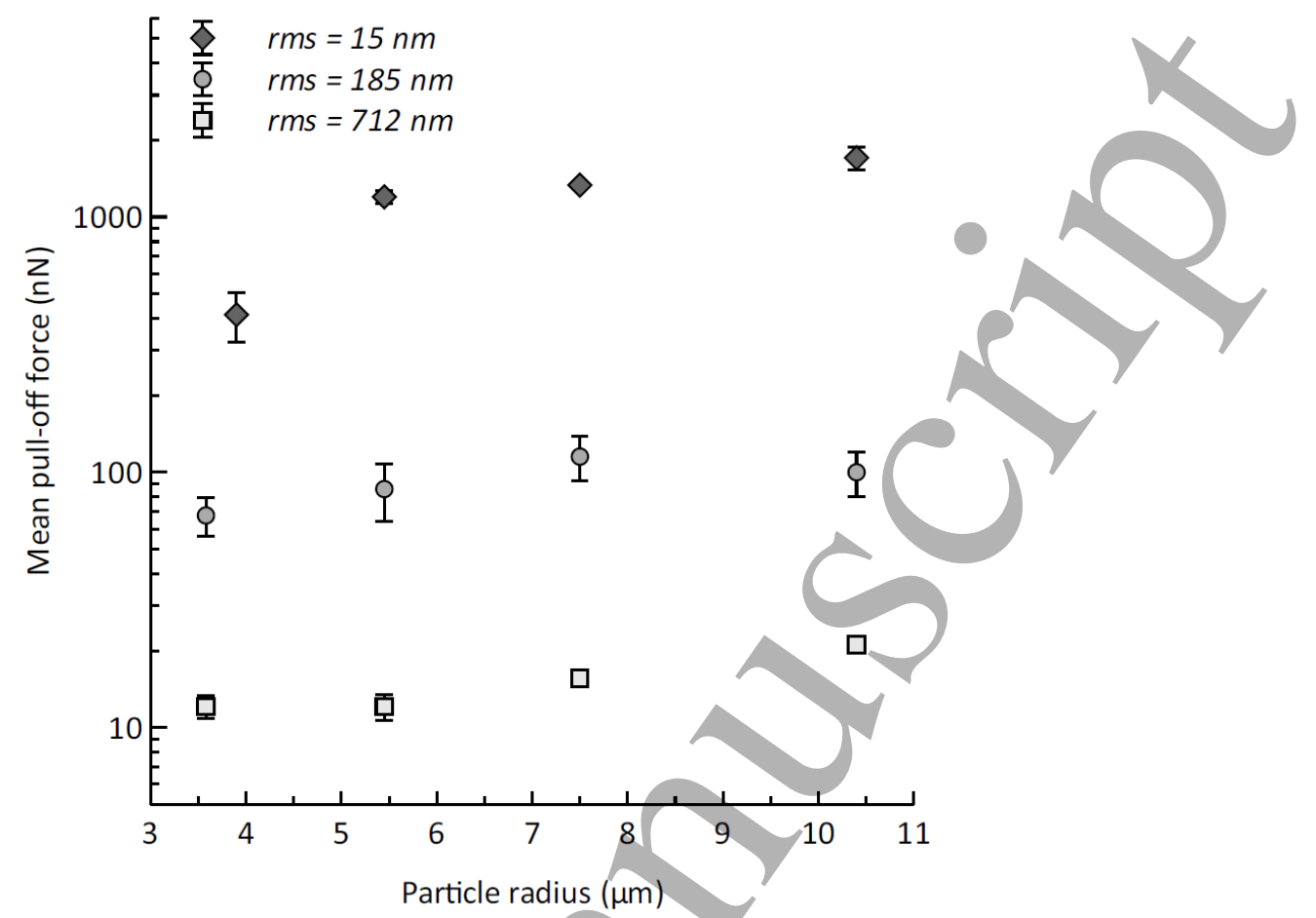

FIG. 5. Adhesion forces obtained by AFM for W spherical particles of different radius in contact with $W$ surfaces of variable roughness. 


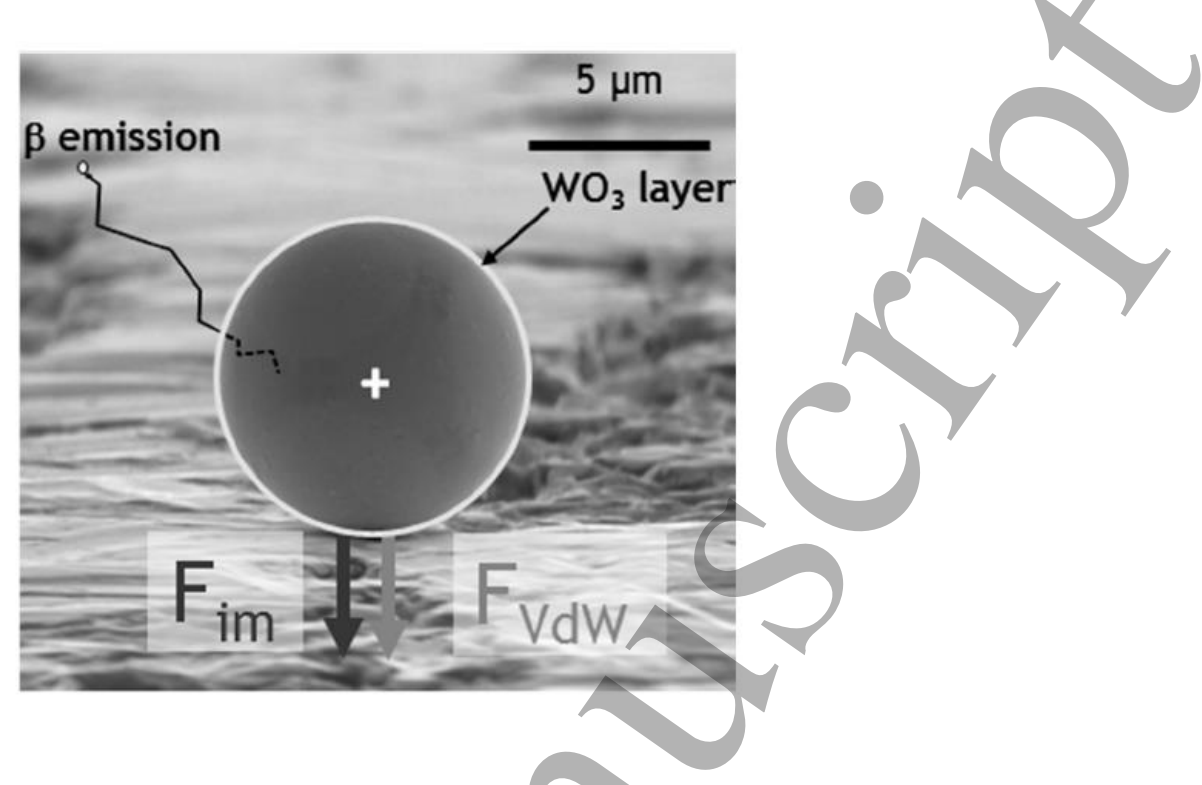

FIG. 6. Schematic of balance of forces in the case of a W spherical tritiated particle lying on a W surface 


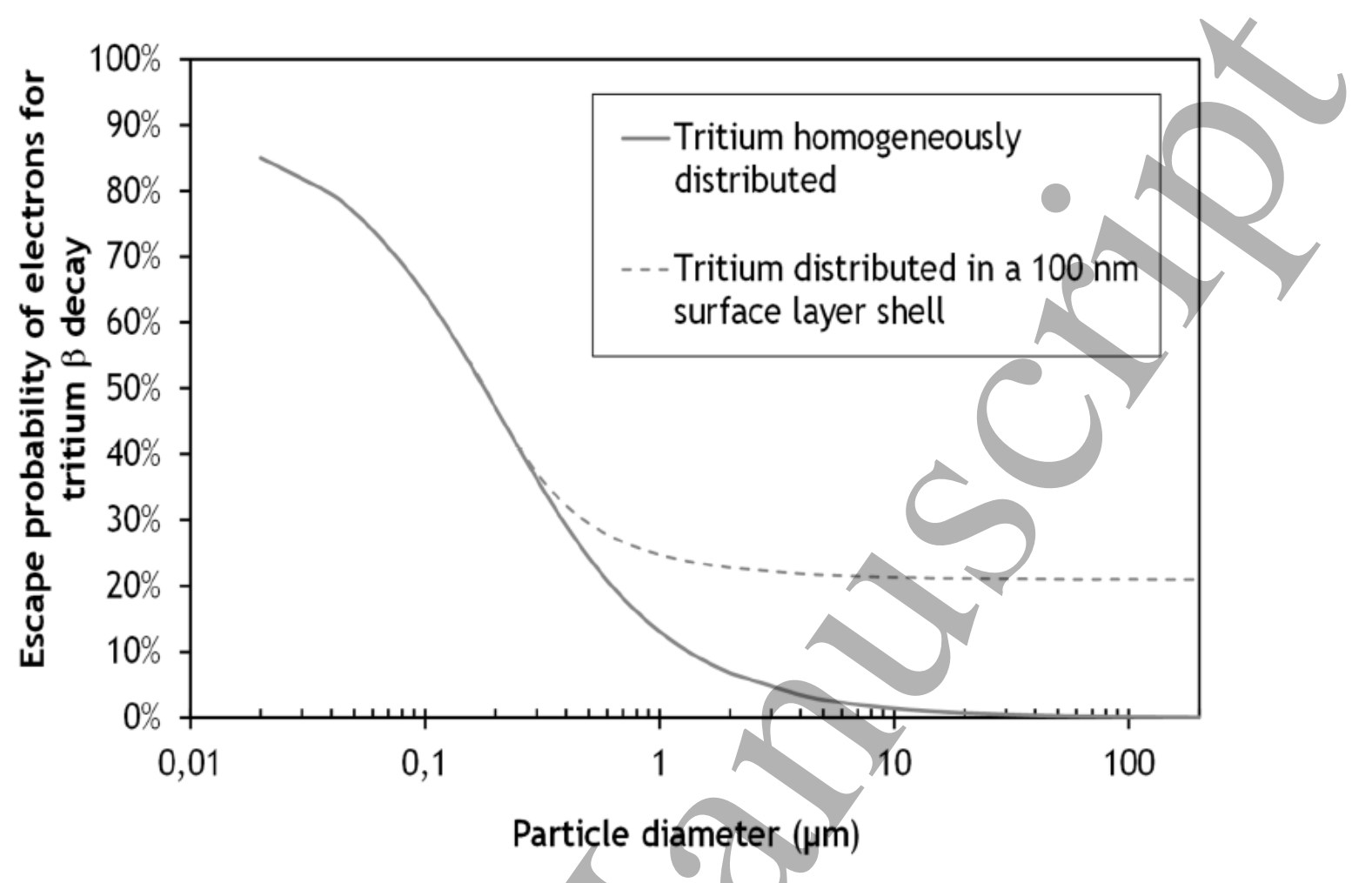

.FIG. 7. Calculation of the escape probability of $\beta$ electrons from $W$ tritiated particles versus the size of the particles for the 2 considered configurations. 


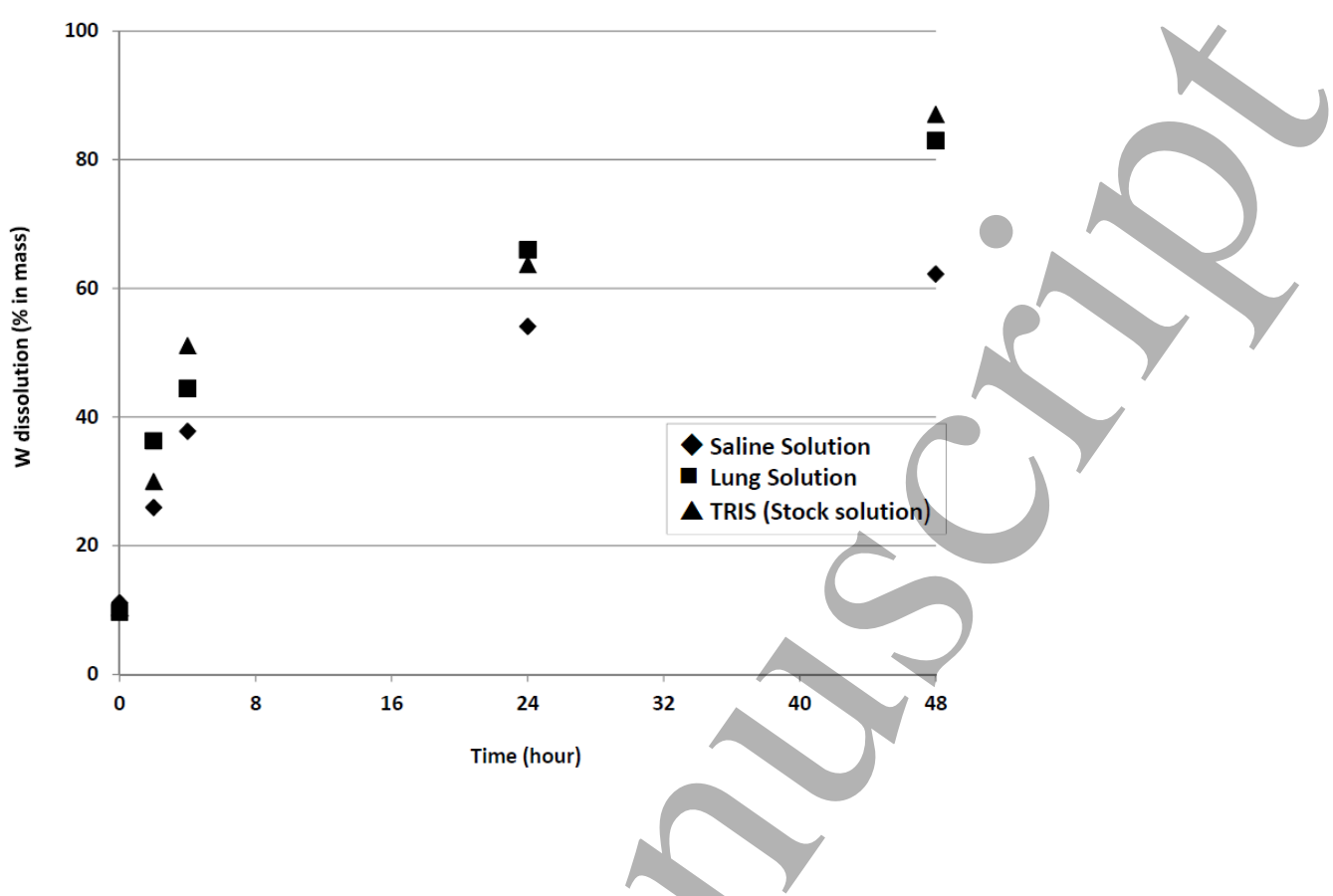

FIG. 8. Dissolution of $100 \mathrm{~nm}$ tungsten particles in different biologic media solutions. 